\title{
Monitoring Physiological Variables With Membrane Probes
}

FINAL REPORT

NAGW 4525

Principal Investigator: Elsa M. Janle, Ph.D

Bioanalytical Systems, Inc. 2701 Kent Avenue West Lafayette, IN 47906

Phone: 317-463-4527

Fax: 317-497-1102 E-Mail: ejanle @bioanalytical.com 


\section{FINAL REPORT \\ NAGW 4525 \\ Monitoring Physiological Variables With Membrane Probes \\ Principal Investigator: Elsa M. Janle, Ph.D}

The microgravity conditions of space flight present the human body with an abnormal environment. Responses to this altered environment can result in many physiological changes. In many cases, these changes are undesirable and development of counter measures will enhance the well being and productivity of humans in space. Study of physiology and development of counter measures may be hampered by the small number of human subjects in space to study, and the undesirability of carrying out invasive procedures on human subjects. Use of animal subjects also presents certain problems. There are a limited number of subjects and a limited number of some types of samples because of the small size of the animal

In this project, we address some of the difficulties inherent in space physiological research involving chemical measurements. Traditionally, the body fluids available for studying chemical changes have been blood and urine. One of the major limitations in using blood, especially in small animals, is the limit of samples which can be taken and the possibility of altering the physiology by removing large volumes of blood. Additionally, blood chemistry represents an average of changes in the entire body. It does not allow for characterizing specific changes within different tissues. The sampling devices and techniques that were developed in this project offer solutions to a number of these problems. The sampling devices used were membrane probes. The probes in this project were placed in subcutaneous and muscle tissue, however, membrane probes can be developed for sampling any tissue. Two different types of probes were used which operate either on the principle of ultrafiltration or microdialysis. The microdialysis probe does not remove any liquid from the tissue but only chemicals which diffuse into the probe. The ultrafiltration probe removes only interstitial fluid which is more easily replaced by the body than blood. These probes have an additional advantage where they can be coupled with on-line sensors to provide real-time data acquisition. In this project, we developed and tested two of these sensors: lactate and glucose.

Although many physiological systems and areas of space medicine could be investigated using these devices and sampling techniques, two areas were explored in this project: electrolytes affected by microgravity and the nutrients glucose and lactate which are important in muscle metabolism.

The methods developed in this project not only have the potential for facilitating both ground based and in flight space research physiology, but may also be used to investigate and clinically monitor many human diseases unrelated to space flight.

\section{PROBE DEVELOPMENT}

Both ultrafiltration (UF) and microdialysis (MD) probes were tested first in vitro and then in vivo. The ultrafiltration probe consists of 3 hollow polyacrylonitrile (PAN) dialysis fibers, each $12 \mathrm{~cm}$ long (OD $320 \mu \mathrm{m}$, ID $220 \mu \mathrm{m}$ ). The thick wall of the fibers is necessary in order to prevent collapse of the fiber under negative pressure sampling conditions. The fibers are formed into a loop and attached to 17" of FEP microbore outflow tubing (OD $0.65 \mathrm{~mm}, \mathrm{ID} 0.20 \mathrm{~mm}$ ) via a short $(1 \mathrm{~cm})$ piece of polyurethane connecting tubing (OD $1.5 \mathrm{~mm}$, ID $0.89 \mathrm{~mm}$ ). The microbore tubing can either be connected to a mini-peristaltic pump or inserted into a hub assembly for use with a Vacutainer ${ }^{\text {TM }}$ (Figure 1A). In this study, the Vacutainer system was used so that UF and MD samples could be collected simultaneously. Two configurations of 
microdialysis (MD) probes have been constructed for implantation in two different locations. For subcutaneous implantation, a loop form (DL) has been used so that implantation can be done with one small incision. The same PAN membrane used in the UF probe was used in the MD probes. For the DL probe, five $\mathrm{cm}$ of membrane was used to maximize recovery while maintaining ultrafiltration losses at an acceptable level (Figure 1B). In this probe, each end of the fiber was attached to 6" of FEP microbore tubing (OD $0.65 \mathrm{~mm}$, ID $0.125 \mathrm{~mm}$ ) using the same polyurethane connecting tubing used in the UF probe. In order to maintain a patent fluid path when the fiber was bent into the loop configuration a 0.003 " 316 stainless steel wire was placed in the lumen of the probe. For sampling from muscle, a linear microdialysis probe design was developed. This probe has a smaller profile than the DL probe to enable it to be threaded though muscle tissue with a minimum of tissue damage (Figure $1 \mathrm{C}$ ). For this probe, $1 \mathrm{~cm}$ of membrane was used. A piece of polyimide tubing (OD 0.216 , ID $0.180 \mathrm{~mm}$ ) was inserted into the lumen of the PAN fiber and glued in place. A $102 \mu \mathrm{m}$ polyester fiber was threaded through the lumen of the probe. It extended beyond the end of the polyimide tubing and was glued at one end occluding the tubing. The function of the polyester fiber was to prevent kinking of the fiber and as an aid for implantation. During implantation the polyester fiber was threaded through a needle which was inserted through the muscle. The fiber was then pulled through the tissue. The occlusion at the end of the tubing prevented tissue from entering and plugging the tubing. After placement, the fiber was threaded through a $1 \mathrm{~cm}$ piece of PEEK tubing designed to increase the diameter of the tubing to the standard probe tubing size of $.65 \mathrm{~mm}$. This was glued to polyimide tubing with a few mm of the polyimide tubing protruding. The occluded end and fiber are cut off with a razor blade or scalpel creating the flow-through path. A piece of this diameter-increasing tubing was attached to the distal end before implantation, since this end did not need to go through the tissue.

A.

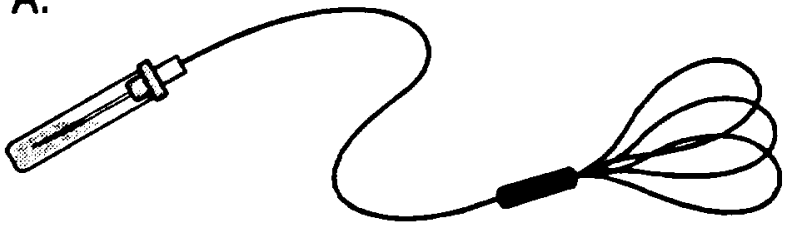

B.

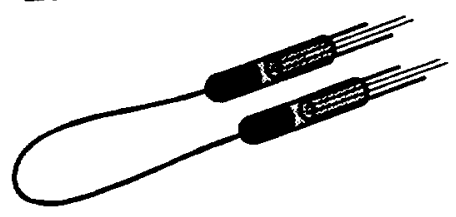

C.

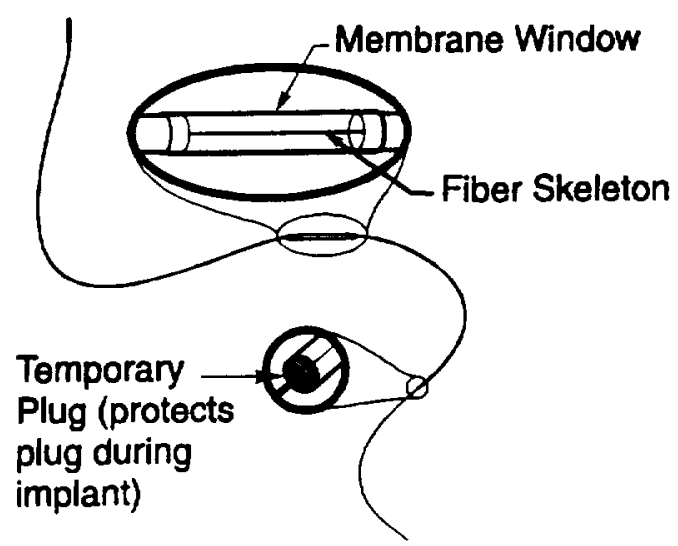

Figure 1. The ultrafiltration probe consists of 3 hollow fibers attached to microbore tubing which can be combined with a hub assembly for Vacutainer sampling (A). The DL microdialysis probe (B) was used for subcutaneous sampling, while the linear microdialysis probe $(C)$ was used for sampling from muscle. 


\section{IN VITRO RECOVERY}

In vitro recovery is the first test of probe acceptability. Recovery refers to the amount of analyte obtained through the probe membrane. There are two measures of recovery: Absolute Recovery and Relative Recovery. Absolute recovery is the total amount of material removed from the system through the probe. Relative Recovery is the concentration of the analyte in the solution obtained from the out flow of the probe relative to the concentration in the solution or tissue being sampled. It is expressed as a percent. It is the relative recovery which was determined in these in vitro tests. In order to provide valid data, the recovery of an analyte with a probe must be great enough to provide a sample concentration which falls within the assay sensitivity limits. In order to provide valid physiological data, the probe must have the same recovery over the range of physiological and pathological concentrations being investigated.

Recovery is affected by a number of factors. Microdialysis recovery depends on membrane surface area, chemical and physical characteristics of the membrane, temperature, the perfusion rate, sample matrix, and chemistry of the analyte. Factors affecting ultrafiltration recovery are membrane characteristics, temperature, sample matrix and chemistry of the analyte. Because of the sample matrix effect which is present in both UF and MD sampling, it is not possible to directly extrapolate in vitro recoveries to in vivo results. However, performance of in vitro recovery studies prior to in vivo studies are important to validate that the analyte crosses the membrane and that there is no interaction of the analyte with the probe materials. One can also get an indication of the concentrations to be expected and the sensitivity of the analytical methods which will be needed.

All recoveries were done in an in vitro recovery station which was developed and constructed for this and another NASA study (Figure 2). Since temperature affects the probe recovery, in vitro studies should be done at the temperature at which the probes will ultimately be used. For in vivo studies this is usually $37^{\circ}$ C. In order to prevent development of concentration gradients during the study, the solution should be stirred. The in vitro recovery station had a heating, stirring unit with a well large enough to hold a $20 \mathrm{~mL}$ scintillation vial. This container is a convenient size for in vitro recovery studies. The container is small enough so that, if expensive analytes are being tested, excessive amounts are not required. It is large enough so that concentration changes in MD and volume changes in UF do not significantly affect results. The temperature of the in vitro recovery station is controlled by a BAS LC-22C Temperature Controller.

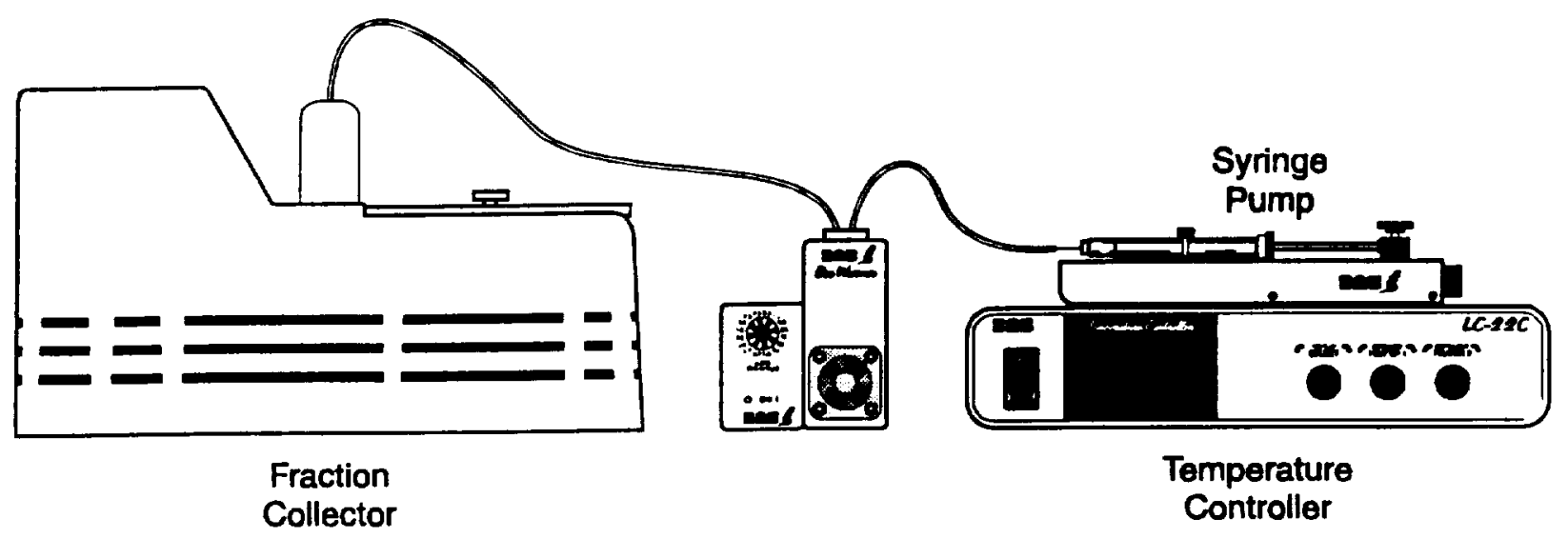

Figure 2. The in vitro recovery apparatus provides temperature control and stirring for the test solution. 
In this study, in vitro recovery was determined for sodium, potassium, chloride, glucose and lactate in ultrafiltration. These are the analytes which were measured in the in vivo studies. Recovery studies were done with ultrafiltration probes with three $12-\mathrm{cm}$ fibers and DL microdialysis probes with $5 \mathrm{~cm}$ membrane lengths. These are the same probes which were used in the in vivo studies. All studies were done in stirred solutions at $37^{\circ} \mathrm{C}$. Three concentrations of each analyte were tested. These concentrations represent the range of possible normal physiological values and pathologically high and low values which might be encountered in the in vivo studies. Table I gives the concentrations used in this study.

Table I. Test concentrations for in vitro recovery.

\begin{tabular}{|l|l|l|l|}
\hline Analyte & Low & Normal & High \\
\hline Sodium & $1.10 \mathrm{meq} / \mathrm{L}$ & $140 \mathrm{meg} / \mathrm{L}$ & $180 \mathrm{meg} / \mathrm{L}$ \\
\hline Potassium & $2 \mathrm{meg} / \mathrm{L}$ & $4 \mathrm{meg} / \mathrm{L}$ & $7 \mathrm{meg} / \mathrm{L}$ \\
\hline Chloride & $70 \mathrm{meg} / \mathrm{L}$ & $100 \mathrm{meg} / \mathrm{L}$ & $130 \mathrm{meq} / \mathrm{L}$ \\
\hline Lactate & $1 \mathrm{mM}$ & $5 \mathrm{mM}$ & $10 \mathrm{mM}$ \\
\hline Glucose & $30 \mathrm{mg} / \mathrm{dL}$ & $100 \mathrm{mg} / \mathrm{dL}$ & $400 \mathrm{mg} / \mathrm{dL}$ \\
\hline
\end{tabular}

Probes were presoaked in water overnight to wash off the glycerin coating. Three probes are tested in each solution. The probes were then placed in fresh, distilled water in the recovery apparatus and held in place with tubing clips. The UF probe was attached to the mini-pump (MF-5200) and pumped at a flow rate of approximately $300 \mu \mathrm{L} / \mathrm{hr}$. Perfusate was pumped through the DL probe using the BAS microdialysis syringe pump (MF-1001) and variable flow rate controller (MF-1020) at a rate of $2 \mu \mathrm{l} / \mathrm{min}$. For electrolyte recovery studies, the perfusate was nano-pure water. For metabolites, the perfusate was Ringers' solution.

The probes were flushed for one hour and the collected samples were used as blanks. The probes were then placed in the test solution. Four one-hour samples were collected. Test solution samples were collected before the start of the recovery study and after each probe sample. Since the first sample was diluted by the dead volume liquid from the flush samples, 2 to 4 were used to calculate recovery.

For each probe in each solution, the recoveries were calculated by dividing the concentration of the probe sample by the average of the test solutions obtained immediately before and after the probe sample. This compensated for any possible changes in test solution concentration due to evaporative losses or unequal solvent and solute removal.

Figure 3 shows the recoveries for one UF probe in the three different glucose test solutions. The solution labeled zero is the flush solution. The concentration of the analyte in this solution should be zero. If a nonzero concentration were obtained it would indicate analyte carry over from a pervious study. Recoveries from the first sample can sometimes appear low because of rinse solution left over in the dead volume of the probe and tubing when the probe is switched from the flush to the test solution. Variations of recoveries in samples 2 to 4 should be only random variations due to variability in assay method. Any significant differences between samples 2 to 4 would indicate interaction of the test compound with probe materials. Interactions of test compounds and probe materials could add a bias to the results. 


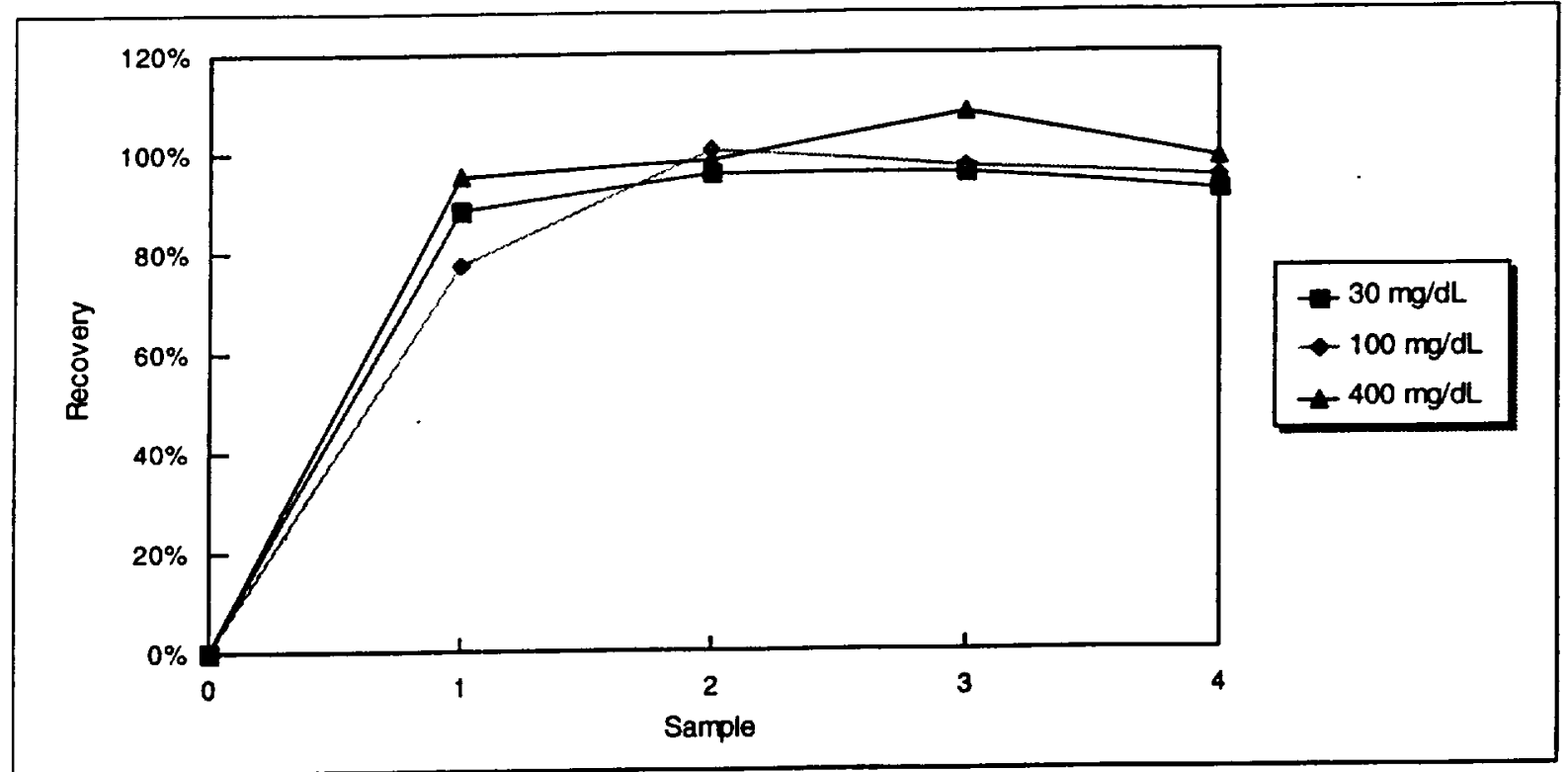

Figure 3. Recovery of one UF probe in solutions of three different glucose concentrations. Sample zero is in saline solution with no glucose. At sample 1 the probe is placed into the test solution. Samples 2 to 4 are used to calculate recovery for the probe.

It is desirable, but not absolutely necessary, that probe recoveries be the same in all probes of a given type. Recovery studies can be done on individual probes before their intended use, but if one can be confident that in vitro recoveries of all probes are the same, this step can be eliminated.

It is also necessary that recoveries be the same for a concentration range which spans the physiological and pathological concentrations to be sampled during in vivo tests with these probes. Figure 4 shows the sodium recovery for each UF probe for each concentration tested.

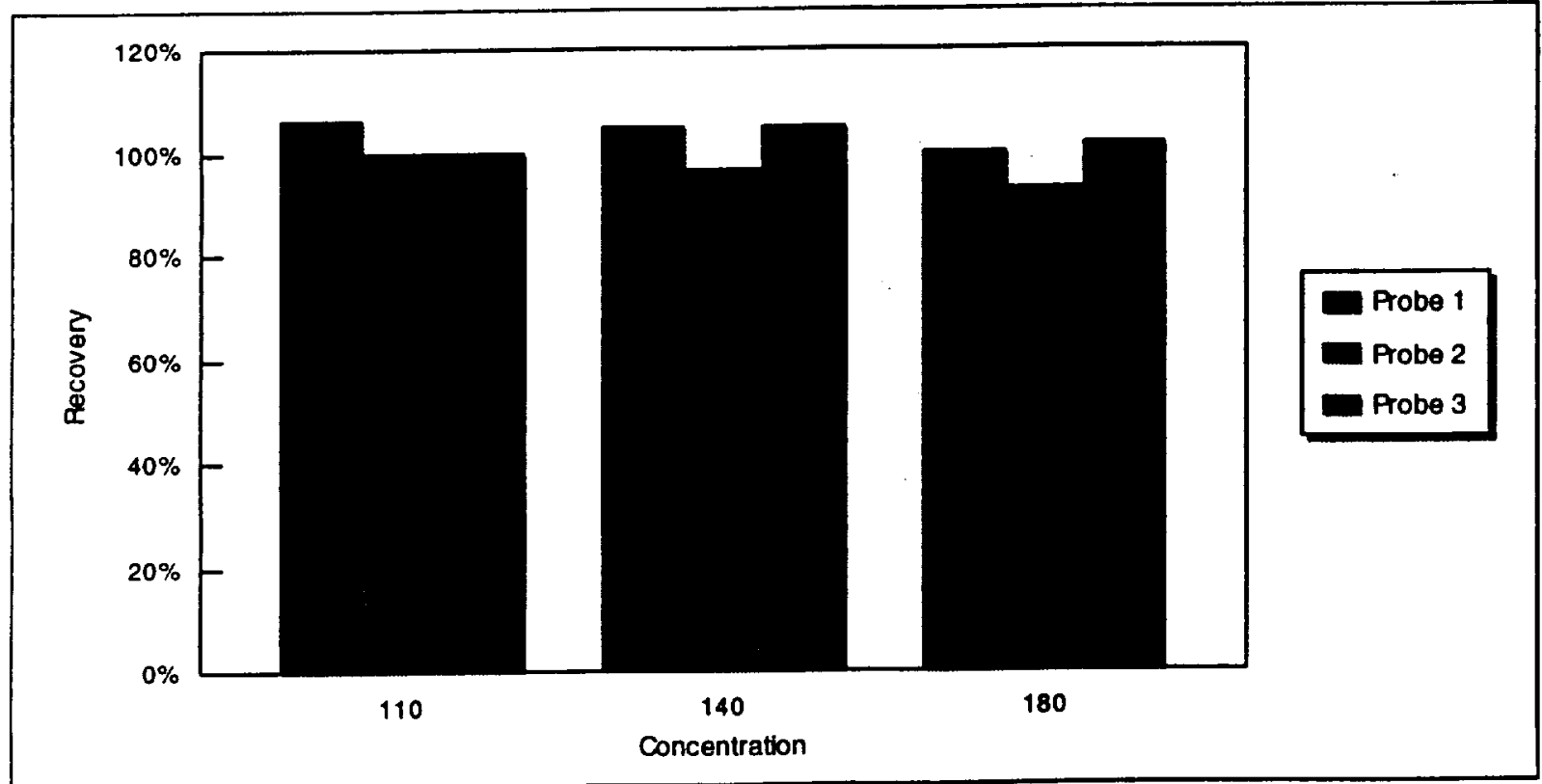

Figure 4. Sodium recoveries for three UF probes at three concentrations spanning the range of physiological and pathological concentrations. Recoveries are the same, within experimental error, for each probe at each concentration. 
For each analyte, two-way ANOVAs without replication $(\alpha=5 \%)$ were done at each concentration on eachprobe by sample, and on each probe for sample number by concentration. For sodium, glucose, and lactate there was no significant effect of sample, probe, or concentration for ultrafiltration or microdialysis probes at the $p=0.1$ level. For potassium, there was no significant effect of concentration or sample for ultrafiltration or microdialysis probes, but there was a significant difference between probes. For chloride, there was no significant effect of any of the factors in the analysis of the UF probes. For the MD probes. there was no significant effect of sample or probe, but there was a significant effect of concentration.

Table II gives the overall recoveries for each probe type for each analyte tested.

Table II: In vitro recoveries.

\begin{tabular}{|l|c|c|}
\hline Analyte & UF-3-12 & DL-5 \\
\hline Sodium & $101 \% \pm 2 \%$ & $101 \% \pm 2 \%$ \\
\hline Potassium & $94 \% \pm 13 \%$ & $106 \% \pm 4 \%$ \\
\hline Chloride & $96 \% \pm 4 \%$ & $95 \% \pm 7 \%$ \\
\hline Glucose & $99 \% \pm 3 \%$ & $90 \% \pm 10 \%$ \\
\hline Lactate & $94 \% \pm 5 \%$ & $95 \% \pm 7 \%$ \\
\hline
\end{tabular}

These recoveries verified that the UF and MD probes were suitable for sampling these analytes in in vivo studies.

One of the goals of this project, which was not completed, was to validate the use of UF and MD probes to measure the certain hormones involved in water and electrolyte balance. The hormones selected were Vasopressin, Aldosterone and ACTH. The recovery studies were done, but when the samples were assayed the concentrations in both the probes samples and the original samples were low. These samples were analyzed with commercial RIA kits from Diagnostic Systems Laboratories. Further work traced the problem to a matrix effect of our samples. The assays were developed for plasma samples and MD and UF samples having a different matrix. Developing assay methods for hormones in MD and UF samples will need to be done as a separate research project. We are currently working with Diagnostic Systems Laboratories to develop these methods and will submit separate proposals to accomplish the task.

Although valid concentrations could not be obtained from microdialysate and ultrafiltrate samples in the recovery studies, the recovery could be calculated by relating the percent bound of the recovery samples with that of the original solution. Using this method, the recovery of vasopressin in UF probes is estimated to be about $91 \%$. The recovery of aldosterone from UF probes was $96 \%$ and from MD probes was $26 \%$. Since these recoveries are high these probes will be able to be used to investigate hormone changes when a RIA for ultrafiltrate and microdialysate samples has been developed.

\section{ULTRAFILTRATION LOSSES FROM MICRODIALYSIS PROBES}

Ideally, with the use of microdialysis probes there is no loss or gain in fluid volume of the perfusate passing through the probe and the sample production rate is equal to the perfusion rate. In practice, there are often changes in fluid volume. Water is a small molecule and can pass through the membrane if there is a driving force. Loss of water from the perfusate to the tissue is referred to as ultrafiltration losses. The most important factor controlling transfer of fluid from the probe to the tissue is a pressure differential. This pressure differential is increased with increased flow rate and increased length of outflow tubing. Ultrafiltration losses are also related to membrane length. The longer the membrane length, the greater the ultrafiltration losses. Longer membrane lengths are frequently chosen to take advantage of the higher 
recoveries obtainable with larger probes. However, the advantages must be balanced with the disadvantage of greater ultrafiltration losses. Characterization of a microdialysis probe involves evaluation of ultrafiltration losses in both in vitro and in vivo studies.

The DL-5 microdialysis probes were tested for ultrafiltration losses during the recovery studies. Weighed vials were used for the collection of samples. Perfusion rates were set at $2 \mu \mathrm{L} / \mathrm{min}$. Individual probes were used from one to five days. Overall ultrafiltration losses were $35.4 \% \pm 13.6 \%$. The data was analyzed by single factor ANOVA to determine if there were significant differences between probes and/or days of use. There was a significant difference in UF losses between probes (Table III), but the differences between days was not significant.

Table III. ANOVA: Single Factor: Days

\begin{tabular}{|c|r|r|r|r|}
\hline SUMMARY & & & & \\
\hline Groups (Days) & \multicolumn{1}{|c|}{ Count } & Sum & Average & Variance \\
\hline 1 & 24 & 8.643 & 0.360125 & 0.024488 \\
\hline 2 & 17 & 5.99 & 0.352353 & 0.017045 \\
\hline 3 & 16 & 5.512 & 0.3445 & 0.016297 \\
\hline 4 & 2 & 0.713 & 0.3565 & 0.010805 \\
\hline 5 & 2 & 0.736 & 0.368 & 0.022898 \\
\hline
\end{tabular}

\begin{tabular}{|l|c|c|c|c|c|c|}
\hline ANOVA & & & & & & \\
\hline Source of Variation & SS & df & MS & F & P-value & F crit \\
\hline Between Groups & 0.002795 & 4 & 0.000699 & 0.035123 & 0.997569 & 2.536581 \\
\hline Within Groups & 1.114093 & 56 & 0.019895 & & & \\
\hline Total & 1.116888 & 60 & & & & \\
\hline
\end{tabular}

ANOVA: Single Factor: Probes

\begin{tabular}{|l|c|r|r|r|r|c|}
\hline ANOVA & & & & & & \\
\hline Source of Variation & SS & df & MS & F & P-value & F crit \\
\hline Between Groups & 0.757985 & 24 & 0.031583 & 3.167921 & 0.00088 & 1.824212 \\
\hline Within Groups & 0.358903 & 36 & 0.00997 & & & \\
\hline & & & & & & \\
\hline Total & 1.116888 & 60 & & & & \\
\hline
\end{tabular}

In vivo ultrafiltration losses were determined in three rats during the hind-limb suspension studies. Twohour samples were collected in weighed vials using a fraction collector. The flow rate was $2 \mu \mathrm{L} / \mathrm{min}$. The studies ranged from 2 to 25 days. The average in vivo ultrafiltration loss was $39 \% \pm 8 \%$. Data was analyzed by ANOVA. Slight differences between studies was obtained (Table IV). There were no significant changes in ultrafiltration losses over time (Table V). 
Table IV. ANOVA: Single Factor: Suspension studies

\begin{tabular}{|c|r|r|r|r|}
\hline SUMMARY & & & & \\
\hline Groups & Count & \multicolumn{1}{|c|}{ Sum } & Average & Variance \\
\hline NASA 9 & 2 & 0.61 & 0.305 & 0.00125 \\
\hline NASA 10 & 16 & 6.78 & 0.42375 & 0.006745 \\
\hline NASA 11 & 15 & 5.41 & 0.360667 & 0.003307 \\
\hline
\end{tabular}

\begin{tabular}{|l|c|c|c|c|c|c|}
\hline ANOVA & & & & & & \\
\hline Source of Variation & SS & df & MS & F & P-value & F crit \\
\hline Between Groups & 0.045433 & 2 & 0.022717 & 4.582473 & 0.01834 & 3.31583 \\
\hline Within Groups & 0.148718 & 30 & 0.004957 & & & \\
\hline Total & 0.194152 & 32 & & & & \\
\hline
\end{tabular}

Table V. ANOVA: Single Factor: Time

\begin{tabular}{|l|r|r|r|r|r|c|}
\hline ANOVA & & & & & & \\
\hline Source of Variation & \multicolumn{1}{|c|}{ SS } & df & MS & F & P-value & F crit \\
\hline Between Groups & 0.064027 & 22 & 0.00291 & 0.203787 & 0.998584 & 3.131277 \\
\hline Within Groups & 0.11425 & 8 & 0.014281 & & & \\
\hline Total & 0.178277 & 30 & & & & \\
\hline
\end{tabular}

\section{ANALYTE ANALYSIS}

Sodium was initially analyzed with the Cardy Compact Ion Meter (Horiba, Japan) for sodium (C-122) and potassium was initially analyzed with the Cardy Compact Ion Meter for potassium (C131). These meters were found to be lacking in the sensitivity necessary to observe the small changes in concentrations which occur in living animals. Therefore, the alternative analytical procedures were investigated.

For sodium, a commercial combination ion selective electrode (MI-425, Microelectrodes, INC.) was used with a voltmeter and a high impedance microelectrode adapter. This setup gave the needed sensitivity. For potassium, a commercial ion selective electrode (MI-442, Microelectrodes, INC.) and reference electrode (MI-402, Microelectrodes, INC.) with the same voltmeter and adapter setup as for sodium were used. This system did not prove to be a stable system. The problem was traced to the reference electrode which was filled with $3 \mathrm{M} \mathrm{KCL}$. Since the samples were measured on the order of 10 to $20 \mu \mathrm{L}$, leakage from the reference electrode was sufficient to continuously change the concentration of the sample resulting in an unstable reading. The problem was solved by filling the reference electrode with $3 \mathrm{M} \mathrm{LiCl}$.

Chloride was analyzed spectrophotometrically using the Sigma Kit 461-3. A commercial ISE for chloride was purchased from Lazar Research to correlate chemically-determined concentrations with electrodedetermined concentrations and to begin development of on-line determination. Lactate was analyzed using the Sigma Kit 735-10. Glucose was analyzed by the BAS LC method using a glucose oxidase immobilized enzyme reactor and a "wired" peroxidase electrode $(1,2)$. 


\section{EFFECT OF SIMULATED MICROGRAVITY ON ELECTROLYTES AND METABOLITES IN THE RODENT MODEL.}

In order to simulate the fluid shifts of microgravity, a suspension system based on the BAS awake animal system was developed for suspending the rats in a head down position. Rats were implanted with a DL-5 microdialysis probe, a UF-3-12 ultrafiltration probe, and a jugular catheter. The rats were placed in the awake animal system and allowed to recover from anesthesia. Samples were collected from a baseline control period of at least 3 days. Control periods varied because an attempt was made to begin all suspensions at the beginning of a work week. This allowed for greater observation and possible adjustments to the system at the beginning of the suspension. The rats were placed in a jacket and suspended for two weeks. (In some cases, the suspension time was shortened because the rats were doing poorly.) The suspension was then followed by a one week recovery period. Nine rats were used for this study.

Probe and Catheter implantation. Rats were anesthetized with a mixture of Ketamine and Xylazine. Microdialysis and ultrafiltration subcutaneous probes were implanted as previously described (3). For the implantation of linear muscle probes, a $1.5 \mathrm{~cm}$ incision was made in the skin of the hind leg, exposing the muscle. The polyester internal fiber of the probe was inserted through the eye of a straight needle. The needle was inserted through the muscle and the probe pulled into the muscle until the membrane window was within the muscle tissue. Both ends of the probe were tunneled subcutaneously and exited at the base of the neck. The incisions were closed and the occluded end of the probe was cut off forming a patent fluid path.

Jugular catheters were implanted by isolating the jugular vein, making an incision into the jugular, inserting the catheter, and suturing around the vessel and catheter. The catheter was then tunneled subcutaneously to exit at the back of the neck close to the exit site of the UF and MD probes. The catheter was tested for patency, filled with the filling solution, and capped. A collar, with a tether line attached, is placed on the rat, and the rat was placed in the awake animal system.

Catheter Development. Various modifications to the jugular catheter have been tried to develop long-term vascular access. A polyurethane catheter with a heparin-lock $(150 \mathrm{U} / \mathrm{mL})$ can be maintained patent for 1 day. Increasing the heparin concentration to $500 \mathrm{U} / \mathrm{mL}$ increased the patency to 7 days. Since the loss of patency is probably due to the diffusion of the heparin lock out of the catheter and subsequent clot formation, a less diffusible filling solution was tried. Filling the polyurethane catheter with Burr's solution (4), a 9:1 glycerin:heparin mixture resulted in patency of 3 to 8 days. The disadvantage of this solution is that it must be withdrawn from the catheter since the final concentration of heparin is $1000 \mathrm{U} / \mathrm{mL}$ the solution. If blockage occurs, the catheter cannot be flushed to restore patency as it can with lower heparin concentrations. In an attempt to develop a catheter, which will function for a longer period of time, we tried working with a company, BSI, which coats catheters with heparin for human use. They prepared for us 4 FEP, silicone, and polyurethane (Micro-Renathane $($ ) heparin-coated catheters each. The FEP catheters proved unsuitable because of lack of flexibility. The silicone catheters, when filled with saline, had a patency of 3 to 4 days, which was not an improvement over non-coated catheters. When the filling solution of the heparin-coated silicone catheter was changed to the Burr's solution, a patency of 17 days was obtained in one catheter but only one day in another catheter. (In the 17 day case, the rat gained access to the catheter and chewed it, so additional duration of patency may have been possible.) The heparincoated Micro-Renathane ${ }^{\circledR}$ polyurethane catheter patency ranged from 4 to 12 days.

Unfortunately, we were not able to pursue this avenue of catheter development further. BSI decided that development of catheters for rats was of too small a commercial potential that they were unwilling to prepare any additional catheters at any cost. 
Awake Animal System. The awake animal system (5) consists of a large round-bottomed containment bowl for the rat and a table that fits over the bowl, which provides a point of attachment for a lever arm with a two-channel swivel. The swivel is attached to the rat and allows the animal to move around freely while providing for sample collection without the twisting of tubing. The perfusion pump is placed on the table above the animal. Microdialysis samples were collected in a refrigerated fraction collector. Microdialysis samples were collected for intervals of 120 minutes. Ultrafiltrate samples were collected with Vacutainers, which were attached to the tether line and changed manually. Ultrafiltrate sample vials were changed morning and evening except when there was a change in condition, such as anesthesia or suspension, in which case the vials were changed when the change in condition occurred.

Suspension System: At the end of the control period the rat was placed in a jacket. Various modifications were tested to develop a secure system from which the animal could not escape. The most successful configuration consisted of a full body jacket with two straps going around the hind legs to prevent escape from the rear. A collar was placed on the animal and the jacket was attached to the collar to prevent escape forward. The tether line was switched from the collar to the rear of the jacket to allow for suspension. The table was raised 4 inches and additional counter weights were added to the lever arm in order to elevate the rear end of the rat. The bowl was replaced with a rectangular container fitted with a wire grid, which provided the rat with a surface to hole, allowing the rat to move around on it's front feet alone (Figure 5).

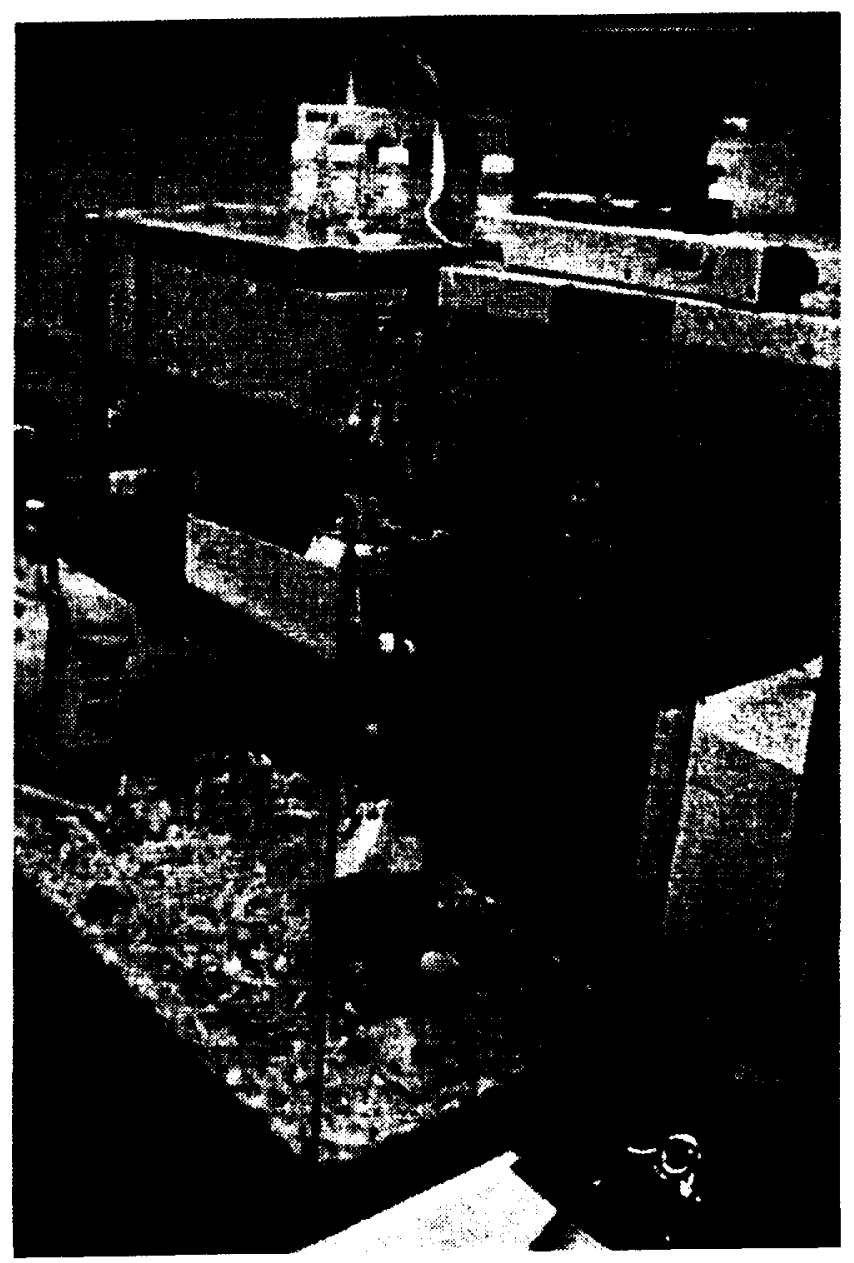

Figure 5. Awake animal suspension system used to simulate microgravity in the rodent model and study effects on electrolytes and metabolites. 


\section{SUBCUTANEOUS ANALYTE CONCENTRATIONS DURING CONTROL AND MICROGRAVITY SIMULATION}

Nine rats were used in the study of effect of microgravity on electrolytes and metabolites. Complete data was not obtained on all rats. Different rats tolerated the stress of suspension differently. Two died during suspension.

Average plasma and subcutaneous concentrations of analytes under different conditions are listed in Table VI. Microdialysis probes are perfused with Ringer's Solution at $2 \mu \mathrm{L} / \mathrm{min}$. Ringer's solution has the following analyte concentrations: sodium, $147 \mathrm{meq} /$; potassium, $4 \mathrm{meq} / \mathrm{L}$; calcium, $4 \mathrm{meq} / \mathrm{L}$; and chloride, $156 \mathrm{meq} / \mathrm{L}$. Ultrafiltrate and microdialysis samples represent average concentrations over the period sampled. Since ultrafiltration and microdialysis sampling are continuous, these concentrations represent averages over the entire sampling period. Plasma concentrations on the other hand are instantaneous values. Plasma values represent averages of single time points.

The probes were implanted under Ketamine-Xylazine anesthesia, which affects some analyte concentrations. Therefore, baseline data collection is begun when the animal has fully recovered from the anesthesia. The data in Table VI represent averages of all rats over the entire period of time under the specified condition. There was, however, considerable variation between animals and there were also changes during the treatments, especially the suspension and recovery periods. Data were analyzed using the t-test to compare concentrations under different conditions. 


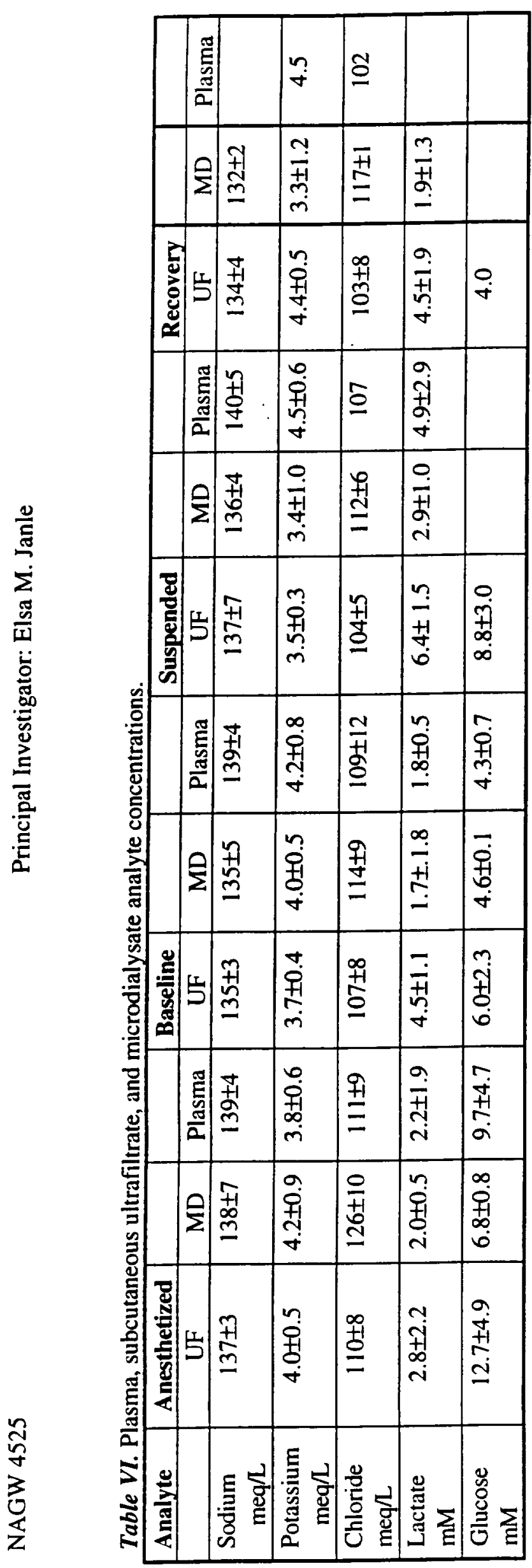


Sodium: In subcutaneous ultrafiltrates, sodium is slightly but significantly higher $(\alpha=.05, \mathrm{p}=.046)$ than baseline during anesthesia. Sodium is also slightly higher than baseline during suspension $(\alpha=.05, \mathrm{p}=$ $.021)$. During the recovery period the sodium decreases to slightly below baseline levels $(\alpha=.05, \mathrm{p}=$ .046). In addition to obtaining average values for analytes under different conditions, the time course of changes of the variables was examined. There is a high degree of variability between animals in some analytes and in the responses to suspension and recovery. More detailed information about responses to anesthesia, suspension, and recovery can be obtained from time courses rather than averages.

Figure 6 shows the time course of ultrafiltrate and plasma sodium changes in a individual rat during baseline, suspension, and recovery. This rat was suspended on day 10 and recovery began on day 19 .

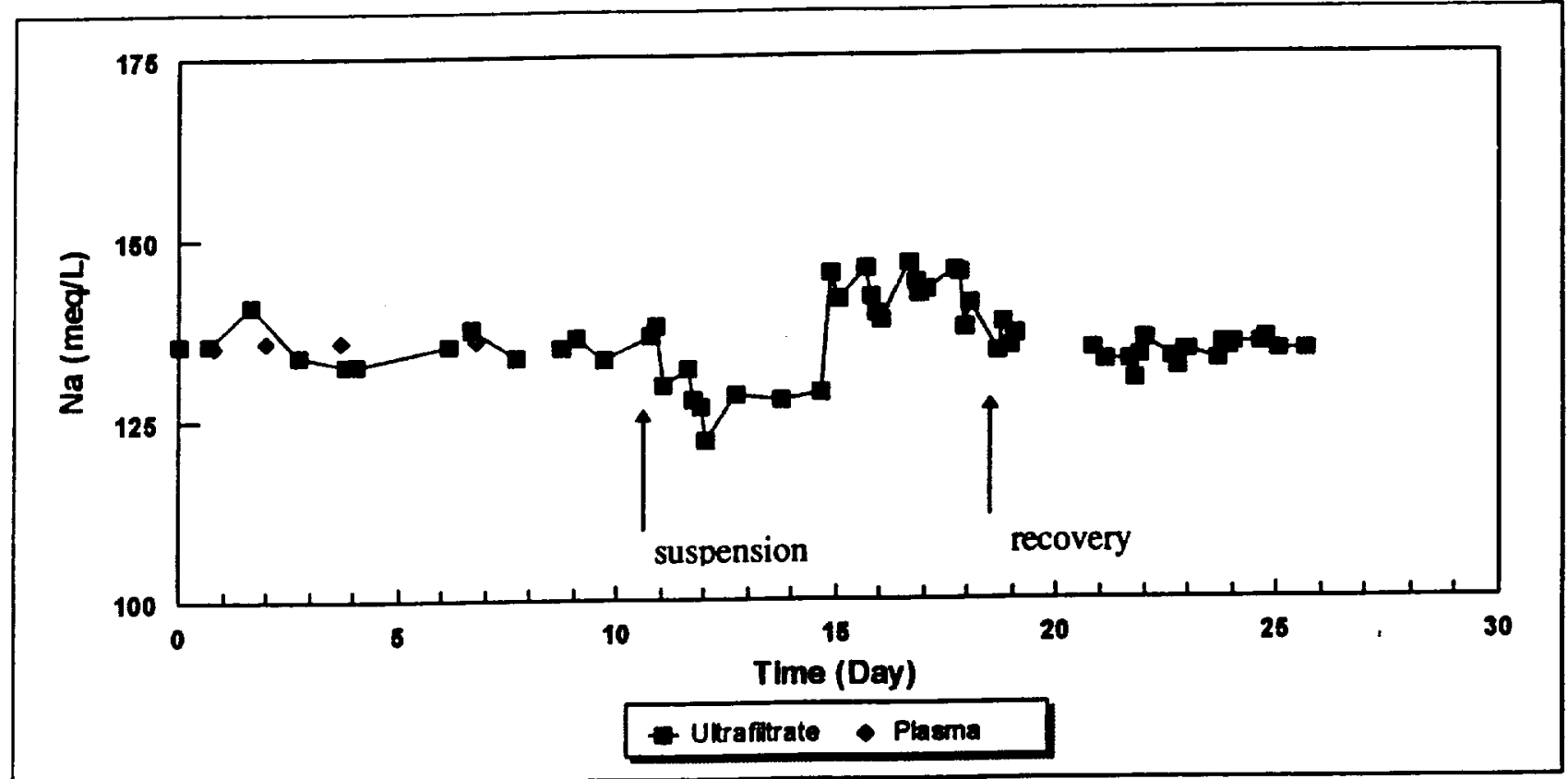

Figure 6. Ultrafiltrate sodium profile of one rat during baseline, suspension, and recovery.

There was minimal effect of anesthesia on sodium. One sample, on the day after anesthesia, was slightly higher, which may have been due to a dehydration effect. Baseline values ranged between 133 and 135 meq/L. After suspension, sodium concentration initially decreased slightly below baseline concentrations for 4 days, then rose above baseline concentrations for 3 days. The concentration returned to baseline levels at the end of the suspension period. During the recovery period, the sodium concentration remained at baseline levels.

The microdialysis averages show the same trends as the ultrafiltrate, with the anesthetized value higher than baseline and the suspended average slightly higher than baseline, and during recovery the sodium levels fell below baseline. Statistical analysis of the microdialysis sodium concentrations did not show significant differences. This is probably due to the fact that there is a greater variation in the microdialysis data because each sample represents a shorter time period and concentrations can vary with water or food consumption throughout the day. In this study the microdialysis samples were obtained over short intervals. It is also possible to obtain ultrafiltration samples over short intervals, but at present, the only reliable swivels available have only 2 liquid channels. Both channels are required for microdialysis sampling, and therefore a choice must be made as to whether to do short interval microdialysis or ultrafiltration sampling. 
Microdialysis was chosen because it is easier to interface with on-line sensors, which were being developed. Figure 7 illustrates the variation in microdialysate sodium concentrations over a baseline period.

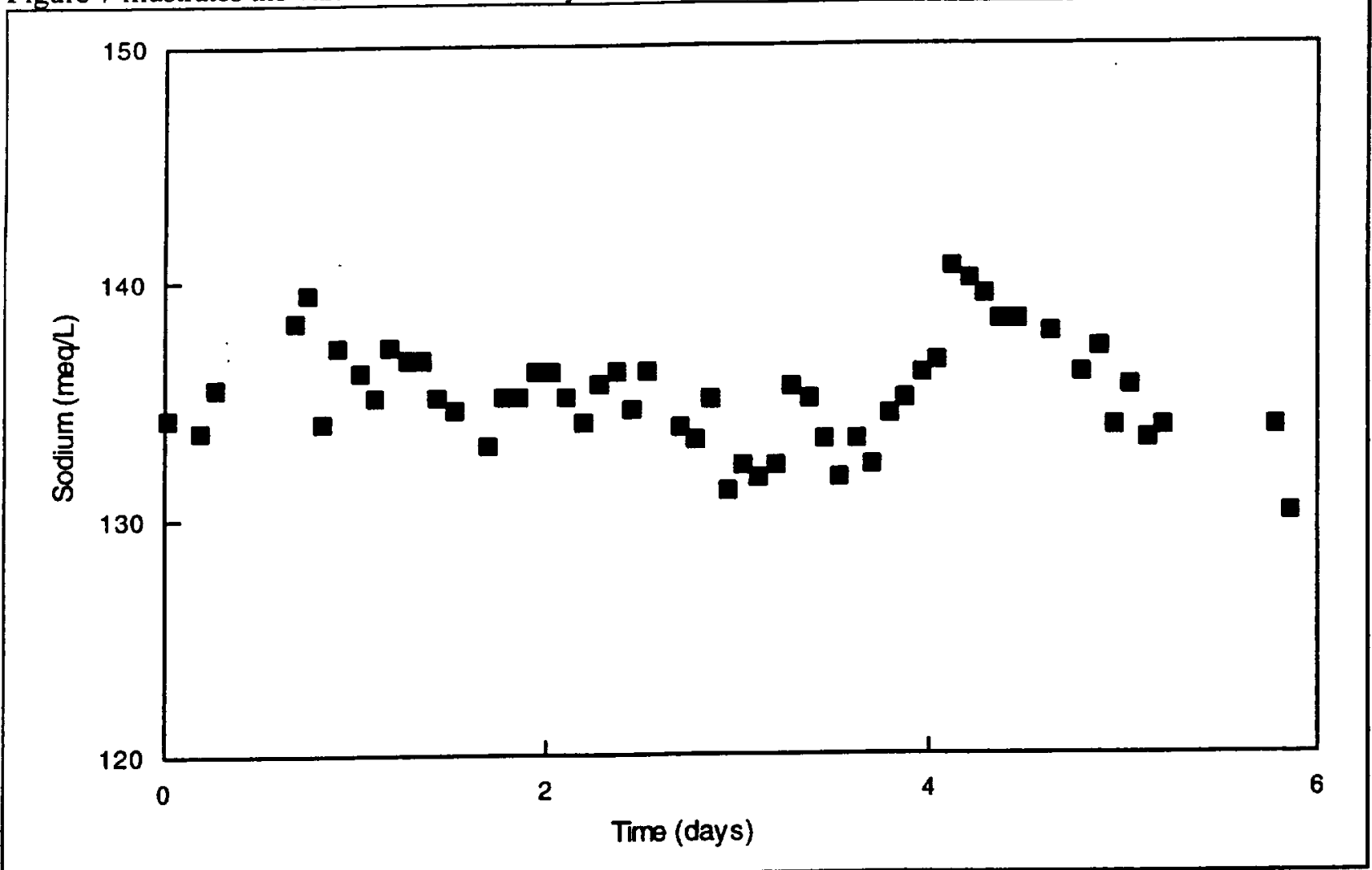

Figure 7. Microdialysis sampling was more frequent than ultrafiltration and therefore gives greater detail of changes. This shows the variation in sodium in one rat during the baseline period.

One type of information available from these probes, which is not available from other sampling methods, is the concentration in different tissues. Sodium concentrations of samples of ultrafiltrate and plasma, taken simultaneously, were compared using a paired t-test. Subcutaneous ultrafiltrate sodium concentrations are significantly lower than plasma sodium concentrations $(\alpha=.05, \mathrm{p}=.013)$. Microdialysis samples also tended to be lower than plasma samples, but the difference was not significant. There were no significant differences between ultrafiltration and microdialysis samples collected simultaneously.

Potassium: Subcutaneous potassium concentrations are slightly higher than baseline values, but the difference is not statistically significant in either microdialysis or ultrafiltrate samples. Suspension results in a decrease in average potassium concentrations. In microdialysis samples the difference is statistically significant $(\alpha=.05, p<0.0000)$. For ultrafiltration samples the difference is not statistically significant. Figure 8 shows the subcutaneous ultrafiltrate in one rat during baseline, suspension, and recovery. In this rat there was an immediate decrease in potassium following head down suspension. The potassium levels began to shift back toward normal levels during the course of suspension, indicating a physiological compensation mechanism was operating. During the recovery period, the concentrations stabilized although concentrations were slightly lower than baseline.

There were insufficient plasma samples for statistical analysis; however, the trends are opposite to subcutaneous concentrations. Plasma potassium is lower than baseline during anesthesia. Suspension resulted in an increase in plasma potassium and there was no change during recovery. 


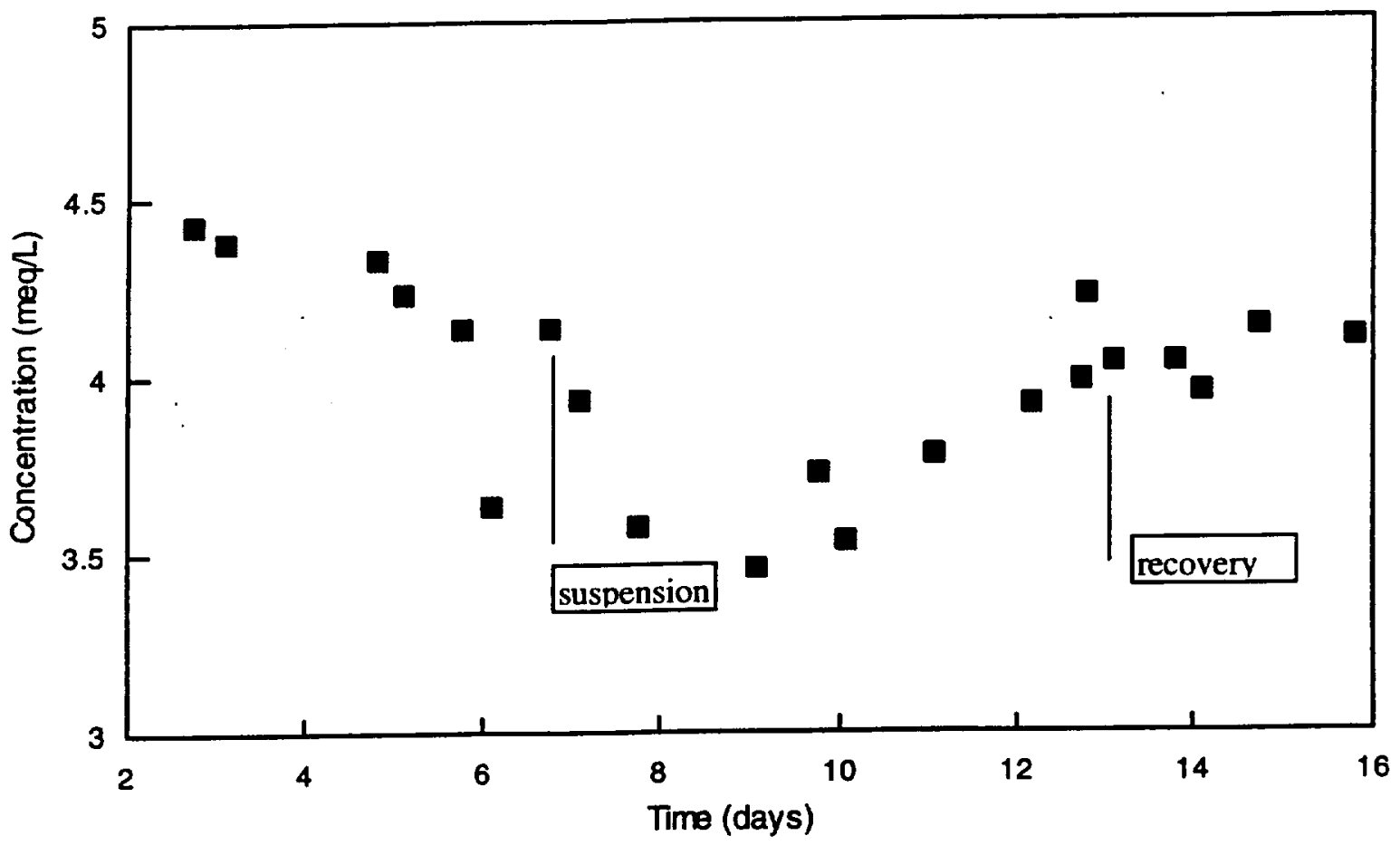

Figure 8. Subcutaneous ultrafiltrate potassium during baseline, head-down suspension, and recovery. Potassium falls after suspension and then begins to retum to normal.

Chloride: Anesthesia causes a slight increase in chloride concentration in subcutaneous tissue and in plasma. Suspension in a head down position results in a decrease in chloride in both subcutaneous microdialysis and ultrafiltration samples while plasma shows a slight elevation. During the recovery period plasma remains unchanged, subcutaneous ultrafiltration showed an increase, and microdialysis a slight decrease.

Lactate and Glucose: Glucose concentrations are increased in both subcutaneous tissue and plasma by anesthesia. Lactate levels are less affected by anesthesia and show a greater variability between animals. The average ultrafiltrate levels were lower, but the plasma and microdialysate levels were slightly higher. Suspension increases the subcutaneous ultrafiltrate glucose levels as well as plasma lactate. During recovery, subcutaneous glucose levels again decrease to baseline levels.

\section{Muscle probe}

Because of the small size of the rat muscle tissue a microdialysis probe must be used for sampling. Since an ultrafiltration probe withdraws all of the fluid from the tissue itself, the volume of sample is limited by the size of the probe. Insufficient sample would be obtained from an ultrafiltration probe to perform the analysis. To sample from the muscle tissue a $1 \mathrm{~cm}$ probe was used. The probe was implanted as described above and perfused with Ringer's solution. Two-hour samples were collected in a refrigerated centrifuge. The samples were analyzed for sodium, chloride, lactate, and glucose. Figure 9 shows the time course of these analytes in one rat under baseline conditions. Analyte concentrations fluctuate considerably under baseline conditions. This may be related to muscle activity. The average level of glucose remains fairly constant through the three day test period. Lactate, however, shows greater fluctuations. This may reflect different levels of lactate production with different levels of activity. The average lactate concentration declines over the course of the implant. Initial higher levels may be due to implant injury. Sodium and 
chloride also show considerable variation. Chloride concentrations are higher than sodium because the chloride concentration of the perfusate is higher than sodium and because of the small membrane surface area equilibrium with the tissue is not reached.
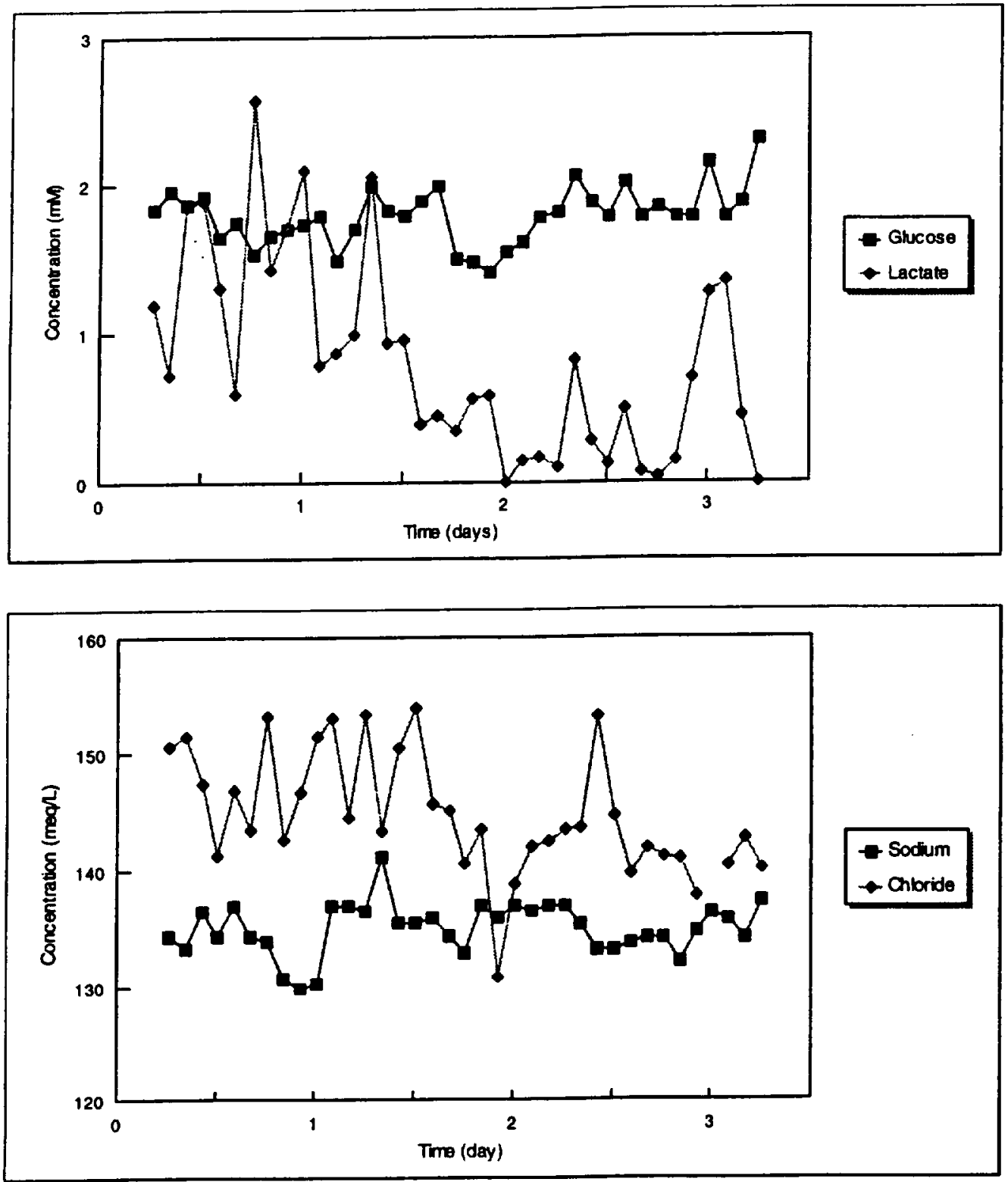

Figure 9. Analyte concentrations in rat muscle tissue. Microdialysis samples were obtained using a $1 \mathrm{~cm}$ linear probe.

These probes demonstrate the possibility of monitoring from specific tissues in small rodents. Additional work will be required to make the probes more durable. They were made with a narrow profile to cause minimum damage, however, this also resulted in probes that were less rugged than the DL type probes. In some cases, the membrane separated from the tubing. In other cases, the delicate nature of the probe tubing resulted in a kink and thus an occlusion of the probe. It is currently possible to get several days of baseline data with these probes, however, the probes cannot withstand the struggles of the rat when placed in a hind leg suspension apparatus. 


\section{DEVELOPMENT OF FLOW CELLS FOR ONLINE IN VIVO MEASUREMENT OF GLUCOSE AND LACTATE}

For the continuous monitoring of glucose or lactate, thin-layer cross-flow amperometric detectors with enzyme modified working electrodes were developed. The enzymes glucose oxidase (GOX) or lactate oxidase (LOX) were covalently immobilized in an osmium redox polymer film on the electrode surface. The inlet and outlet tubing of the amperometric flow cell were compatible with the microdialysis tubing, and the testing conditions for the detector, such as perfusion solution and flow rate, were typical of microdialysis experiments.

The GOX electrode was prepared by coating the electrode surface with the enzyme and polymer mixture solution followed by a cellulose acetate and Nafion over-coating. The over-coating on the enzyme electrode served as mass transport limiting membranes for better linearity. For the in vitro test of the glucose sensor, the amperometric flow cell with the GOX electrode was perfused with standard solutions at $5 \mu \mathrm{L} / \mathrm{min}$. The average $0-90 \%$ rise time of the response was $2 \mathrm{~min}$. The electrode gave a linear response to $0.1-20 \mathrm{mM}$ glucose concentrations, which covers the clinically relevant glucose concentrations. The electrode exhibited poor sensitivity to glucose concentrations below $0.1 \mathrm{mM}$ due to oxygen competition with the redox polymer for GOX. After 72 hours of continuous use, the electrode lost $50 \%$ of its sensitivity. However, the electrode was still usable and the linearity of the response did not change. Oxidase-based electrochemical sensors used for analyses of biological fluid samples often suffer from various interferences such as ascorbic acid, uric acid, and acetaminophen. In this study, the electrooxidation currents for artificial CSF solutions containing $0.5 \mathrm{mM}$ uric acid, $0.1 \mathrm{mM}$ ascorbic acid, or $1 \mathrm{mM}$ acetaminophen were measured with a GOX electrode. The concentrations of these interferences shown above are close to their relevant physiological concentrations except for acetaminophen, which for a worst case might be expected to be about $0.1 \mathrm{mM}$. The solution of uric acid did not show any electrooxidation current. There was an oxidation current of $2 \mathrm{nA}$ for the ascorbic acid solution. This current was equivalent to a $0.1-\mathrm{mM}$ variation in the glucose concentration calculated from the calibration of the electrode. The electrode response to the acetaminophen solution was $60 \mathrm{nA} / \mathrm{mM}$ so that a typical maximum response could be $6 \mathrm{nA}$, which was equivalent to a $0.2-\mathrm{mM}$ variation in the glucose concentration. Since the glucose concentration in most biological samples is much higher than $0.2 \mathrm{mM}$, the interferences from both ascorbic acid and acetaminophen should be tolerable.

The LOX electrodes were prepared similarly. The average $0-90 \%$ rise time of the response was 3 min when the perfusion solution at $5 \mu \mathrm{L} / \mathrm{min}$ was changed between different lactate standards. The electrode gave linear responses to lactate from $0.1 \mathrm{mM}$ to $5 \mathrm{mM}$ and lost $50 \%$ of their sensitivities after $24 \mathrm{hrs}$ of continuous operation. The LOX electrode showed no responses to $0.5 \mathrm{mM}$ uric acid and $0.1 \mathrm{mM}$ ascorbic acid solutions. However, it gave $233 \mathrm{nA} / \mathrm{mM}$ oxidation current for acetaminophen solution. Further efforts are underway in our lab to eliminate the acetaminophen interference in the LOX modified amperometric detector.

Because the cellulose acetate and Nafion overcoating has poor reproducibility, we have recently replaced the overcoating with a polycarbonate dialysis membrane. The dialysis membrane has shown a much better reproducibility of the sensor. It also showed a better selectivity against interferents. We have also explored the use of a disposable printed carbon electrode for the sensor development. Printed carbon electrodes have many advantages over the conventional glassy carbon electrodes. These include low cost, mass production, better quality control and disposablability. 


\section{In Vivo Sensor Tests}

For testing the on-line sensors, DL subcutaneous probes were used because of their durability. The system used for in vivo sensor tests was similar to previously described microdialysis systems, except that a liquid switch was incorporated so that the sensor could periodically be switched from the animal to calibrating solutions.

Sensors were calibrated daily before being connected to the animal. Sensors lost about $25 \%$ of activity over a four day period. In order to increase analyte concentrations, intraperitoneal injections of glucose and lactate were used. To decrease glucose levels, intraperitoneal injections of insulin were used. Figure 10 shows the output of the lactate sensor during baseline conditions and after injection of lactate. Figure 11 shows the glucose concentrations determined from the sensor output during a baseline period, after injection of glucose to raise glucose levels, and after injection of insulin to lower glucose levels.

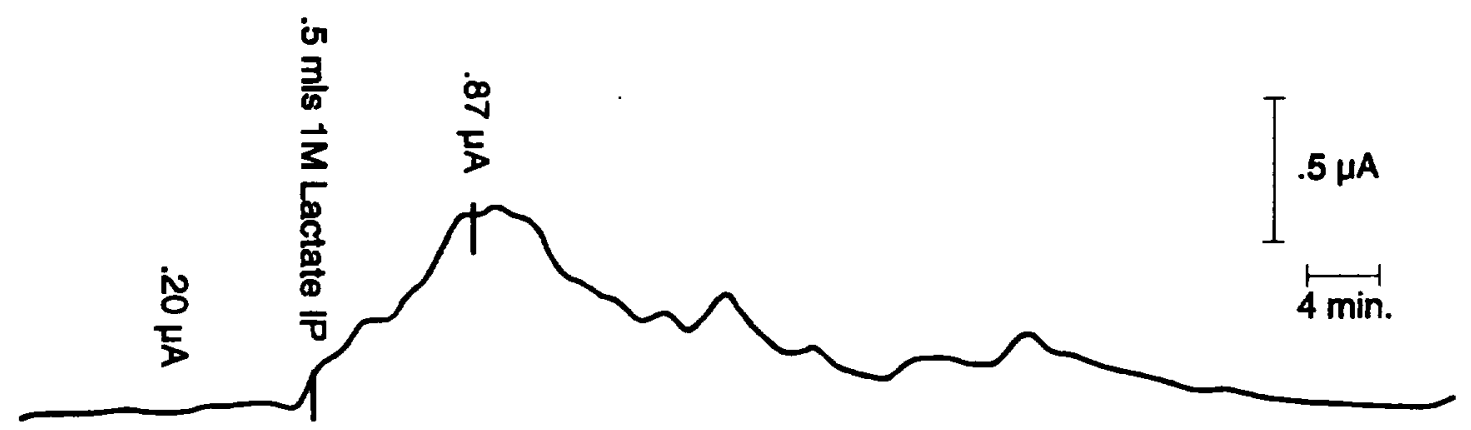

Figure 10. Lactate sensor output during a subcutaneous DL probe during baseline conditions and after intraperitoneal lactate injection.

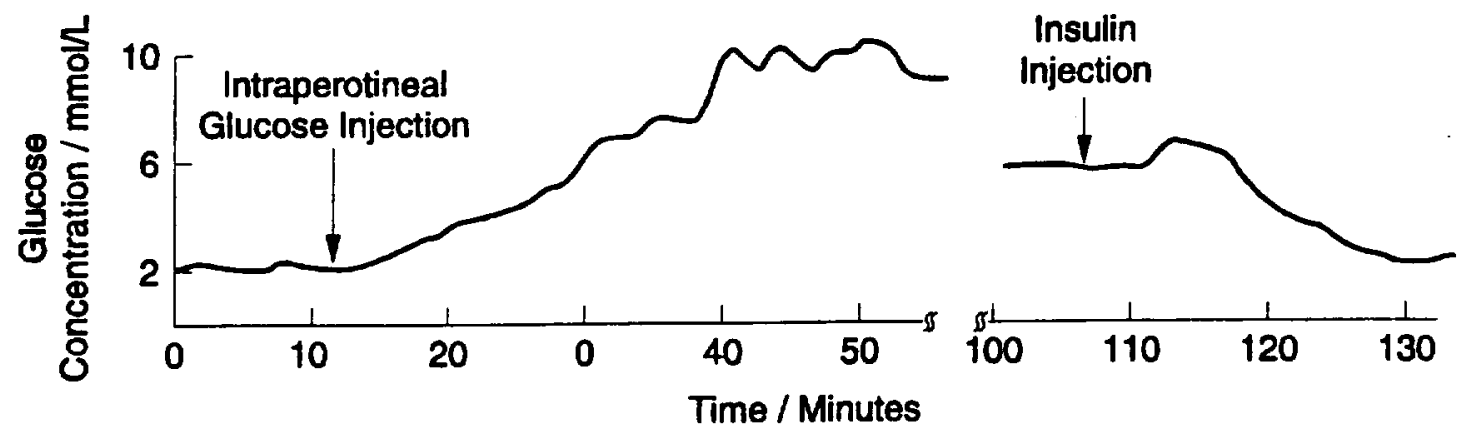

Figure 11. Glucose concentrations determined from the output of a sensor coupled to a subcutaneous DL microdialysis probe during a baseline period, after injection of glucose to achieve an elevated glucose level and after injection of insulin to lower glucose levels. 


\section{Summary}

This project has demonstrated the possibility of using membrane probes in rodents to monitor physiological variables for extended periods of time. The utility of these probes in physiological studies of microgravity has also been demonstrated. The feasibility of developing on-line sensors has also been demonstrated and allows for the possibility of developing real-time automated monitoring systems which can be used in ground-base physiological research as well as in research and medical monitoring in space.

In addition to space applications these techniques can be extended to medical monitoring in critical care situations on earth as well as facilitating research in many human and animal diseases.

\section{References}

1. Yang L, Janle E, Huang T, Gitzen J, Kissinger PT, Vreeke M, Heller A: Applications of "Wired" peroxidase electrodes for peroxide determination in liquid chromatography coupled to oxidase immobilized enzyme reactors. Anal Chem 67: 1326-31, 1995.

2. Janle EM, Ash SR, Zopp WE, Kissinger P: Determination of glucose in microliter samples of in vivo ultrafiltrates and microdialysates using amperometric flow injection analysis with an enzymatic reactor. Current Separations 12:14-16, 1993.

3. Janle, EM: Implantation of Subcutaneous Probes in Rodents. Bioanalytical Systems Applications Capsule \#241.

4. Brown DF and Burr RE, Customized Vascular Catheters for Rodents. Laboratory Animal Science, 35 : 515-6, 1985.

5. Gitzen, J, Janle E, Buelher, E and Hampsch J. A Rodent Residence for In Vivo Bioanalytical Sampling. Current Separations 13: 88-89, 1994.

\section{INVENTIONS DEVELOPED UNDER CONTRACT NAGW 4525}

- All devices and techniques developed under this contract were developed by Bioanalytical Systems, Inc. There were no subcontractors.

- Sampling systems for small rodent microgravity simulations utilizing membrane probes.

- On-line lactate and glucose sensors for use with membrane probes.

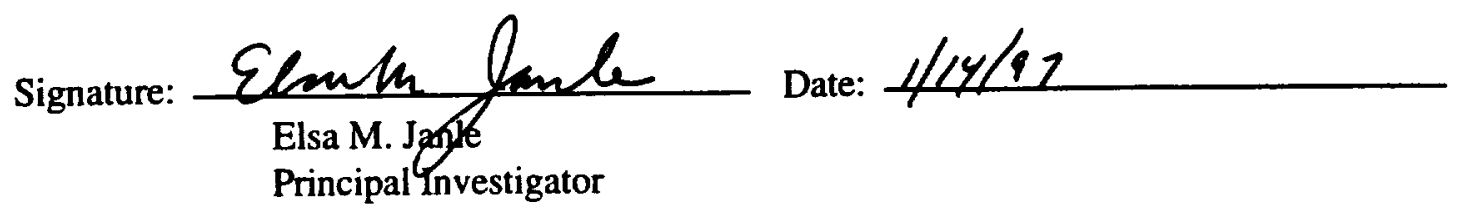

